\title{
Efficacy and safety of IV ferumoxytol for iron deficiency anemia in patients with cancer
}

\author{
Saroj Vadhan-Raj' \\ Naomi V Dahl ${ }^{2}$ \\ Kristine Bernard ${ }^{2}$ \\ Zhu $\mathrm{Li}^{2}$ \\ William E Strauss ${ }^{2}$ \\ 'Division of Cancer Medicine, \\ University of Texas, MD Anderson \\ Cancer Center, Houston, TX, USA; \\ ${ }^{2}$ AMAG Pharmaceuticals, Inc., \\ Waltham, MA, USA
}

This article was published in the following Dove Press journal: Journal of Blood Medicine

\begin{abstract}
Purpose: Iron deficiency anemia (IDA) is common in cancer patients due to blood loss and inflammation. Many do not tolerate oral iron or adequately respond. Intravenous (IV) iron is commonly used as an adjunct to erythropoiesis-stimulating agents; data on the use of IV iron monotherapy in these patients are limited. This study aimed to evaluate IV ferumoxytol for the treatment of cancer patients with IDA with a history of unsatisfactory oral iron therapy or in whom oral iron could not be used.
\end{abstract}

Patients and methods: This post hoc analysis of pooled data from two multicenter, randomized, controlled, Phase III trials evaluating IV ferumoxytol $(510 \mathrm{mg} \times 2)$ vs placebo or iron sucrose (200 mg $\times 5)$ included a subgroup of 98 patients with cancer that the investigator identified as the primary cause of their IDA, or with cancer whose IDA was attributed to another comorbid condition (ferumoxytol, $n=75$; iron sucrose, $n=13$; placebo, $n=10$ ). Gastrointestinal cancers were most common (42), followed by breast (14), cervix (ten), and lung (nine). The primary endpoint was the mean change in hemoglobin (Hgb) from baseline to week 5 .

Results: At week 5, both ferumoxytol and iron sucrose produced significant increases in $\mathrm{Hgb}$ from baseline $(1.8 \mathrm{~g} / \mathrm{dL}[P<0.0001]$ and $1.9 \mathrm{~g} / \mathrm{dL}[P=0.002]$, respectively). During the studies, 45 patients received chemotherapy, 19 with platinum-based regimens. Erythropoiesis-stimulating agent doses were neither increased $>20 \%$ nor initiated in any treatment group. Overall rates of adverse events and serious adverse events in the cancer subgroup mirrored those in the overall study population.

Conclusion: Monotherapy with IV iron appears to be an effective option for cancer patients with IDA who do not respond to or cannot tolerate oral iron therapy.

Keywords: anemia, cancer, ferumoxytol, hemoglobin, efficacy, intravenous iron

\section{Introduction}

The National Comprehensive Cancer Network (NCCN) Guidelines for Cancer- and Chemotherapy-Induced Anemia report that $32 \%-60 \%$ of patients with cancer have iron deficiency. ${ }^{1}$ Anemia is frequently observed as a comorbidity in patients with cancer, and it is estimated that $30 \%-90 \%$ of patients with cancer are also anemic. ${ }^{2}$ Patients with anemia have poorer rates of survival and local tumor control compared with similar non-anemic cancer patients. ${ }^{2}$ The causes of anemia in patients with cancer may be difficult to evaluate as they are often multifactorial and may result from bleeding, nutritional deficiencies, hemolysis, renal insufficiency, hereditary disease, anemia of chronic disease, or a combination of these comorbidities. ${ }^{1,3,4}$ In addition, malignant cancer cells may exacerbate anemia through various mechanisms of action including
Correspondence: Naomi $\vee$ Dahl

AMAG Pharmaceuticals, Inc., II 00

Winter St, Waltham, MA, 0245I, USA

Tel +l 6174987650

Fax +l 6179022420

Email ndahl@amagpharma.com 
direct suppression of hematopoiesis via bone marrow infiltration or cytokine-induced suppression of iron sequestration (ie, cancer-related inflammation). ${ }^{1,5}$ Anemia may also result from anticancer therapies, including antineoplastic drugs (via either direct myelotoxicity ${ }^{4}$ or diminished renal erythropoietin production because of nephrotoxicity [eg, cisplatin]). ${ }^{6,7}$ Newer targeted therapies such as tyrosine kinase inhibitors can also induce anemia, although mechanisms are not fully established. ${ }^{8}$ Possible explanations include an effect on hematopoiesis via FLT3 and c-Kit receptor blockade, chronic bleeding resulting from anti-angiogenic drugs, and thrombotic microangiopathic hemolytic anemia. ${ }^{8}$

Anemia is typically managed by the treatment of its underlying cause, transfusion with packed red blood cells, or the use of erythropoiesis-stimulating agents (ESAs), which may be used in conjunction with iron supplementation. ${ }^{1}$ NCCN guidelines recommend considering supplementation with intravenous (IV; preferred) or oral iron without an ESA for patients with absolute iron deficiency, defined by the NCCN as serum ferritin levels $<30 \mathrm{ng} / \mathrm{mL}$ and transferrin saturation (TSAT) levels $<20 \%$. ${ }^{1}$ If oral iron is used and no response is seen after 4 weeks, a trial of IV iron should be considered. According to the NCCN guidelines, if hemoglobin $(\mathrm{Hgb})$ increases after 4 weeks, then periodic evaluation (repeated ferritin and TSAT testing) is recommended. However, if Hgb does not increase after 4 weeks, then a diagnosis of functional iron deficiency (defined by the NCCN as ferritin 30-800 $\mathrm{ng} / \mathrm{mL}$ and TSAT 20\%-50\%) should be considered. ${ }^{1}$

For patients with functional iron deficiency, NCCN guidelines recommend considering IV iron supplementation as an adjunct to treatment with an ESA. ${ }^{1}$ These guidelines also state that insufficient data exist to routinely recommend IV iron as monotherapy without an ESA for the treatment of functional iron deficiency anemia (IDA). ${ }^{1}$ Data from a metaanalysis performed by Gafter-Gvili et al supports the NCCN recommendation to consider the use of IV iron therapy. A comparison of IV iron with no iron or oral iron for the treatment of chemotherapy-induced anemia (CIA) demonstrated that IV iron significantly increased hematopoietic response rate (RR, 1.28; 95\% CI, 1.125-1.45; seven trials with an ESA) and decreased the rate of blood transfusions both in trials with an ESA (RR, 0.76; 95\% CI, 0.61-0.95; seven trials) and without an ESA (RR, 0.52; 95\% CI, 0.34-0.80; two trials). ${ }^{9}$

Ferumoxytol (Feraheme ${ }^{\circledR}$; AMAG Pharmaceuticals Inc., Waltham, MA, USA) consists of a superparamagnetic iron oxide core stabilized by a semi-synthetic carbohydrate shell, composed of polyglucose sorbitol carboxymethylether (PSC), designed to help isolate the bioactive iron core from plasma components until the iron-PSC complex enters the reticuloendothelial system macrophages. ${ }^{10-12}$ Ferumoxytol was approved in June 2009 by the US Food and Drug Administration for the treatment of IDA in adult patients with chronic kidney disease. ${ }^{13}$ Given the limited data evaluating IV iron monotherapy in patients with CIA or in those with cancer and IDA from any mechanism, we sought to extract efficacy and safety data on the use of ferumoxytol in patients with cancer from two Phase III trials investigating the efficacy and safety of IV ferumoxytol for treatment of IDA in patients with a history of unsatisfactory oral iron therapy or in whom oral iron could not be used. ${ }^{14,15}$ The patient populations in these two studies were stratified into several predefined subgroups based on the patients' primary underlying cause of their IDA, including cancer, as attributed by the investigators. ${ }^{14,15}$ For these prespecified subgroup analyses, the cancer subgroups only included those patients whose cancer was attributed by the investigators as the primary underlying cause of their IDA; thus, the subgroups did not include patients with cancer who had anemia attributed by the investigators to another cause. Here, we report the results from a post hoc subgroup analysis designed to provide information on all of the patients with cancer who were enrolled in these studies. This includes those whose cancer was identified as the primary cause of their IDA as well as other patients with cancer whose IDA was attributed to another condition by the investigators (eg, gastrointestinal bleeding or abnormal uterine bleeding [AUB]) but who also had a diagnosis of cancer.

\section{Material and methods Study design and medication}

The safety and efficacy of IV ferumoxytol for the treatment of IDA in patients with a history of unsatisfactory oral iron therapy or in whom oral iron could not be used were evaluated in two randomized, multicenter, global Phase III studies (ClinicalTrials.gov identifiers: NCT01114139 [Study 1] and NCT01114204 [Study 2]). Study details have been previously published..$^{14,15}$ In brief, Study 1 was a double-blind trial that randomized patients in a 3:1 ratio to receive either parenteral ferumoxytol (1.02 g course) or placebo (Figure 1). ${ }^{15}$ Patients received an IV injection of either ferumoxytol 510 $\mathrm{mg}(17 \mathrm{~mL})$ or normal saline, administered as an IV injection of $17 \mathrm{~mL}$ at a rate not to exceed $1 \mathrm{~mL} / \mathrm{sec}$ (generally in under 1 minute) at baseline (day 1), with a second dose 2-8 days later. Study 2 was an open-label, active-controlled trial, ${ }^{14}$ conducted simultaneously with Study 1, in which patients with the same entry criteria were randomized 2:1 to receive either ferumoxytol, with a second dose 2-8 days later, or iron 


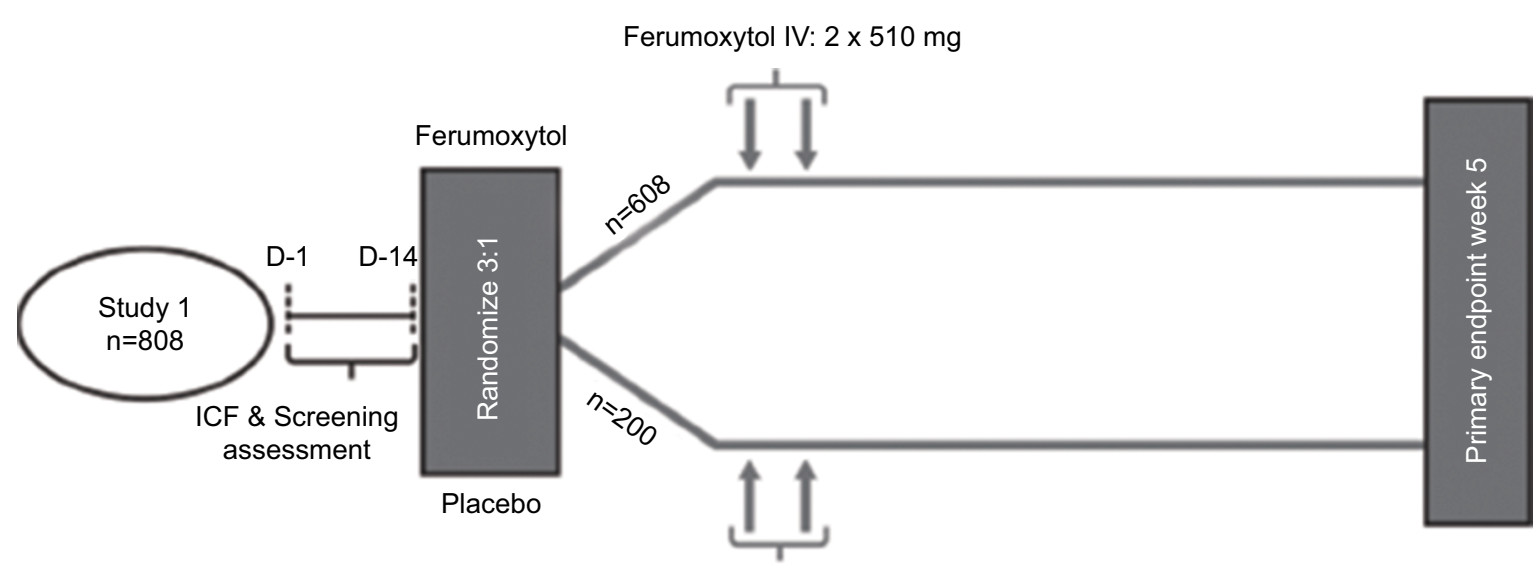

Placebo IV: $\times 2$

Ferumoxytol IV: 2 x $510 \mathrm{mg}$

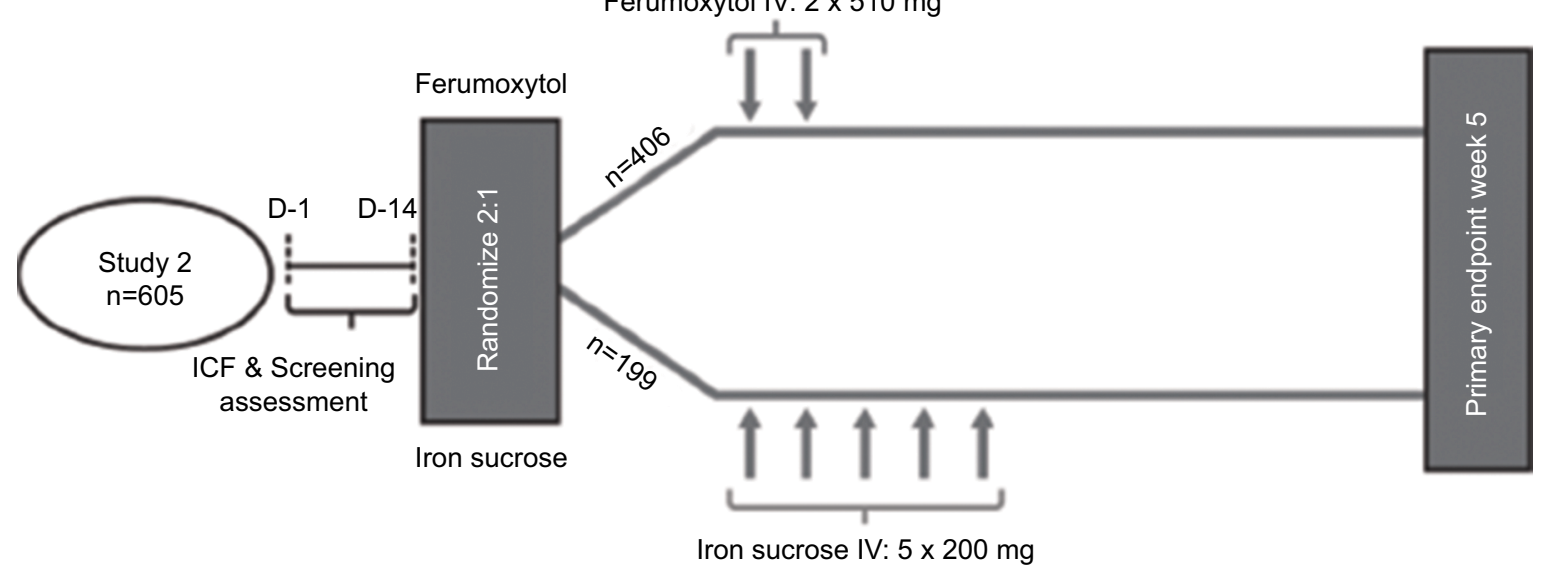

Figure I Study design of Study I and Study 2.

Abbreviations: EP, endpoint; ICF, informed consent; IV, intravenous; D-I, one day prior to randomization; D-I4, I4 days prior to randomization.

sucrose, administered as five infusions or injections of $200 \mathrm{mg}$ on 5 nonconsecutive days over a 14-day period (Figure 1). For both studies, blood sampling to assess Hgb was performed, and the Functional Assessment of Chronic Illness TherapyFatigue (FACIT-F) questionnaire was administered, at screening, baseline, and weeks 2, 3, 4, and 5 . The primary efficacy endpoint was the proportion of patients achieving a $\geq 2 \mathrm{~g} / \mathrm{dL}$ increase in $\mathrm{Hgb}$ at any time from baseline to week 5 .

In both of these studies, the patients' primary underlying cause of IDA was attributed by the investigators to one of five subgroups (cancer, gastrointestinal disorders, AUB, postpartum anemia, or other condition [included patients with nutritional iron deficiency, heart failure, and/or rheumatoid arthritis]). This post hoc analysis includes both patients whose cancer was identified as the primary cause of their IDA as well as other patients with cancer whose IDA was attributed to another condition by the investigators (eg, gastrointestinal bleeding or AUB) but who also had a diagnosis of cancer.

\section{Patient population}

A full description of the inclusion and exclusion criteria has been published previously. ${ }^{14,15}$ Briefly, eligible patients were males and females aged $\geq 18$ years with IDA defined as baseline $\mathrm{Hgb} 7$ to $<10 \mathrm{~g} / \mathrm{dL}$, TSAT $<20 \%$, and history of unsatisfactory oral iron therapy or intolerance to oral iron. Patients were excluded if they had an estimated glomerular filtration rate $<30 \mathrm{~mL} / \mathrm{min} / 1.73 \mathrm{~m}^{2}$, serum ferritin $>600 \mathrm{ng} / \mathrm{mL}$, or history of allergy to IV iron. Written informed consent was obtained from all patients prior to study entry. The study protocols were reviewed and approved by the institutional review boards (IRBs) or ethics committees at each study site (names of each approving IRB and ethics committee are provided in Supplementary material). Both studies were conducted in accordance with the ethical principles of Good Clinical Practice and in compliance with the Declaration of Helsinki.

\section{Assessments and endpoints}

The primary efficacy endpoint of this subgroup analysis was the mean change in Hgb from baseline to week 5. Other efficacy endpoints included the mean change in $\mathrm{Hgb}$ from baseline at earlier time points, receipt of blood transfusion, and changes in patient-reported fatigue. Safety was assessed throughout the 5-week follow-up period and included the overall incidence of treatment-emergent adverse events 
(TEAEs), serious adverse events (SAEs), study drug-related adverse events (AEs) and SAEs, and AEs leading to study drug discontinuation, as well as AEs of special interest (predefined as moderate-to-severe hypotension occurring on the day of dosing and moderate-to-severe hypersensitivity reactions), composite cardiovascular AEs (predefined as nonfatal myocardial infarction, heart failure, moderate-to-severe hypertension, and hospitalization due to any cardiovascular event), and deaths. AEs were assessed by the investigators for severity (mild, moderate, and severe) and potential relationship to study medication. AEs were considered "serious" if they resulted in death, were life-threatening, resulted in hospitalization or persistent or significant disability or incapacity, or were considered an important medical event (ie, one that was not immediately life-threatening but clearly jeopardized the patient and/or required intervention). Laboratory and safety data were pooled for this analysis.

\section{Statistical methods}

The safety population included all randomized patients who had any exposure to the study drug and was based on actual treatment received. The intent-to-treat (ITT) population included any randomized patient who had any exposure to the study drug (IV ferumoxytol, IV iron sucrose, IV saline placebo) and was based upon randomized treatment assignment. The safety and ITT populations were identical in this analysis. Demographics and baseline characteristics were compared between treatment groups using the Fisher exact test for categorical variables (sex, race, type of cancer, history of IV iron use, and Hgb category) and analysis of variance for continuous variables (Hgb, TSAT, and serum ferritin). In the overall study population, changes from baseline in continuous variables were compared between treatment groups using analysis of covariance controlling for baseline $\mathrm{Hgb}$ value, using an imputed value of 0 for change from baseline for missing post-baseline values. In this cancer subgroup analysis, within-group differences in change from baseline $\mathrm{Hgb}$ and FACIT-F were analyzed with a paired $t$-test. The incidence of blood transfusions and use of ESAs were summarized using descriptive statistics.

\section{Results}

\section{Patients}

The ITT population of all 1413 patients who were exposed to the study drug included 98 patients with cancer (ferumoxytol, $n=75$; iron sucrose, $n=13$; placebo, $n=10$ ). Baseline laboratory values and demographic information are shown in Table 1 . The majority of patients in this subgroup
Table I Baseline patient demographics by treatment group

\begin{tabular}{|c|c|c|c|}
\hline \multirow[t]{2}{*}{ Baseline characteristics } & \multicolumn{3}{|c|}{ Treatment group } \\
\hline & $\begin{array}{l}\text { Ferumoxytol } \\
(n=75)\end{array}$ & $\begin{array}{l}\text { Iron } \\
\text { sucrose } \\
(n=\mid 3)\end{array}$ & $\begin{array}{l}\text { Placebo } \\
(n=10)\end{array}$ \\
\hline Age, y, mean (SD) & $58.5(14.1)$ & $66.7(11.3)$ & $53.4(14.0)$ \\
\hline Female, n (\%) & $56(75)$ & $6(46)$ & $8(80)$ \\
\hline Height, cm, mean (SD) & I6I.5 (8.2) & $165.0(10.3)$ & $152.3(5.7)$ \\
\hline Weight, kg, mean (SD) & $68.4(21.3)$ & $73.8(18.8)$ & $55.5(20.4)$ \\
\hline $\begin{array}{l}\text { History of IV iron } \\
\text { exposure, } n(\%)\end{array}$ & $6(8)$ & $2(15)$ & $I(10)$ \\
\hline Hgb level, g/dL, mean (SD) & $9.0(0.8)$ & $9.0(0.9)$ & $8.8(1.1)$ \\
\hline TSAT, \%, mean (SD) & $9.4(10.7)$ & $7.7(3.4)$ & $9.6(7.3)$ \\
\hline $\begin{array}{l}\text { Serum ferritin, } \mathrm{ng} / \mathrm{mL} \text {, } \\
\text { mean (SD) }\end{array}$ & $120.8(195.9)$ & $151.3(189.6)$ & $80.8(93.9)$ \\
\hline \multicolumn{4}{|l|}{ Baseline Hgb level, g/dL, n (\%) } \\
\hline$>7.0$ to $\leq 8.5$ & $22(29)$ & $4(31)$ & $3(30)$ \\
\hline$>8.5$ to $<10.0$ & $53(7 \mathrm{I})$ & $9(69)$ & $7(70)$ \\
\hline \multicolumn{4}{|l|}{ Race, n (\%) } \\
\hline White & $47(63)$ & $13(100)$ & $4(40)$ \\
\hline Black/African American & $6(8)$ & $0(0)$ & $0(0)$ \\
\hline Asian & $22(29)$ & $0(0)$ & $5(50)$ \\
\hline Other/multiracial & $0(0)$ & $0(0)$ & $I(10)$ \\
\hline \multicolumn{4}{|l|}{ Type of cancer, n (\%) } \\
\hline Gastrointestinal & $3 I(4 I)$ & $8(62)$ & $3(30)$ \\
\hline Breast & $12(16)$ & I (8) & $I(10)$ \\
\hline Other & $9(12)$ & $2(15)$ & $0(0)$ \\
\hline Cervix & 7 (9) & $0(0)$ & $3(30)$ \\
\hline Lung & 7 (9) & $0(0)$ & $2(20)$ \\
\hline Bladder & $3(4)$ & I (8) & $0(0)$ \\
\hline Ovarian & $3(4)$ & $0(0)$ & $I(10)$ \\
\hline Pancreas & $2(3)$ & $0(0)$ & $0(0)$ \\
\hline Prostate & $I(I)$ & I (8) & $0(0)$ \\
\hline
\end{tabular}

Notes: No significant difference between groups in sex, race, type of cancer, history of IV iron use, Hgb category (Fisher exact test), baseline TSAT, or ferritin (two sample $t$-test).

Abbreviations: Hgb, hemoglobin; IV, intravenous; TSAT, transferrin saturation.

were female $(71 \%)$ and white $(65 \%)$, with a median age of 60.5 years. Overall, gastrointestinal cancers were the most common (43\%), followed by breast $(14 \%)$, cervix $(10 \%)$, and lung (9\%) (Table 1). During the studies, 45 patients received chemotherapy, including 19 patients with platinumbased regimens, and seven patients received radiotherapy. At baseline, mean TSAT was $9.2 \pm 9.7 \%$ and ferritin was $121 \pm 187 \mathrm{ng} / \mathrm{mL}$, and approximately $30 \%(\mathrm{n}=29)$ of patients had $\mathrm{Hgb}>7.0$ to $\leq 8.5 \mathrm{~g} / \mathrm{dL}$.

\section{Efficacy}

Treatment with both IV ferumoxytol and iron sucrose was associated with a significant increase from baseline in $\mathrm{Hgb}$ by the end of the study (ferumoxytol $1.8 \mathrm{~g} / \mathrm{dL}[P<0.0001]$ [Figure 2A] and iron sucrose $1.9 \mathrm{~g} / \mathrm{dL}[P=0.002]$, paired $t$-tests). Mean increase in $\mathrm{Hgb}$ from baseline to week 5 for the total pooled populations was $2.7 \mathrm{~g} / \mathrm{dL}$ for both ferumoxytol 
A

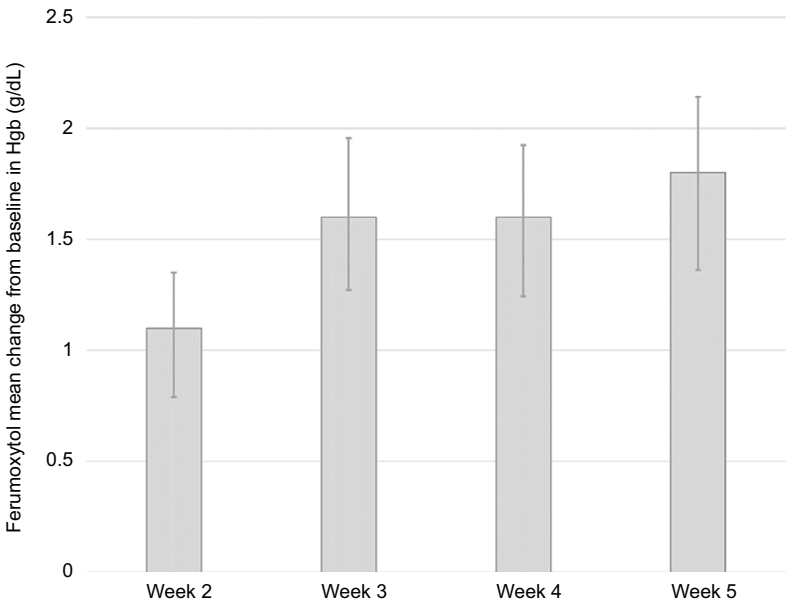

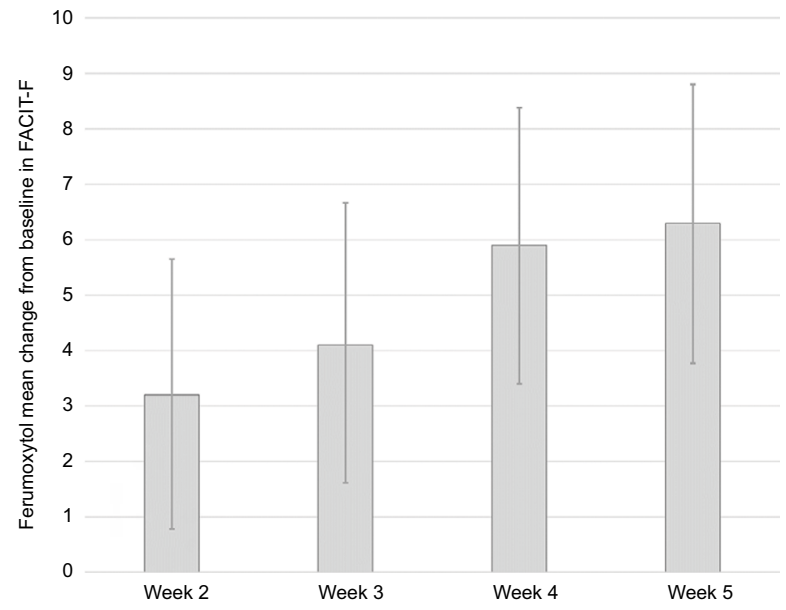

Figure 2 Mean change from baseline hemoglobin (A) and FACIT-F (B).

Notes: Bars represent $95 \% \mathrm{Cls}$, demonstrating within-group changes from baseline.

Abbreviations: Hgb, hemoglobin; FACIT-F, Functional Assessment of Chronic Illness Therapy-Fatigue.

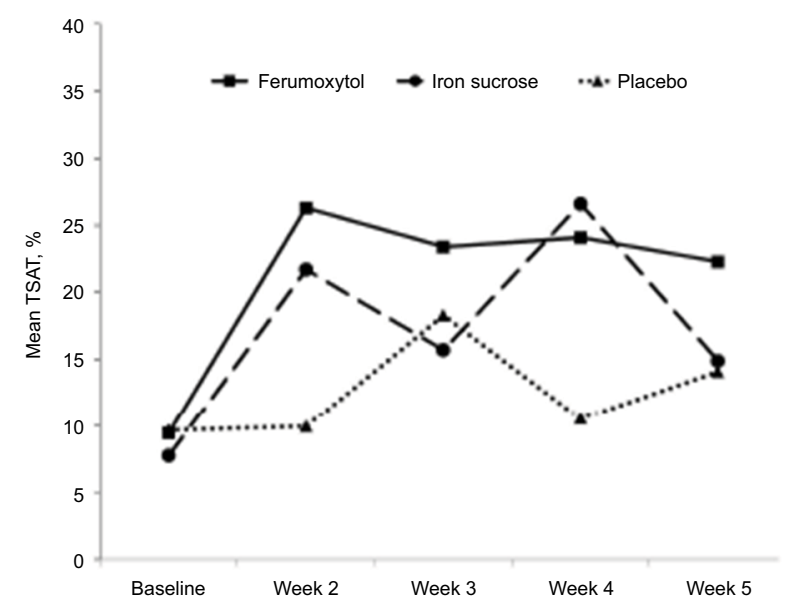

Figure 3 Mean TSAT (A) and median ferritin (B) levels by treatment group. Abbreviation: TSAT, transferrin saturation.

and iron sucrose treatment arms. TSAT and ferritin values also increased over the study period for both IV ferumoxytoland iron sucrose-treated patients with cancer (Figure 3). The changes among patients with cancer were generally similar to those seen for the overall patient populations in these studies for TSAT, while the changes for ferritin were somewhat greater among patients with cancer. Mean TSAT for the total pooled populations in these studies increased from $6.7 \%$ at baseline to $20.9 \%$ at week 5 in the IV ferumoxytol group and from $5.5 \%$ at baseline to $18.0 \%$ at week 5 in the iron sucrose group, compared with a small change for those in the placebo group (5.4\% to $5.8 \%$, respectively). For the total pooled populations, median ferritin levels increased from $4.5 \mathrm{ng} / \mathrm{mL}$ at baseline in both the IV ferumoxytol and iron sucrose groups to $291.0 \mathrm{ng} / \mathrm{mL}$ and $145.0 \mathrm{ng} /$

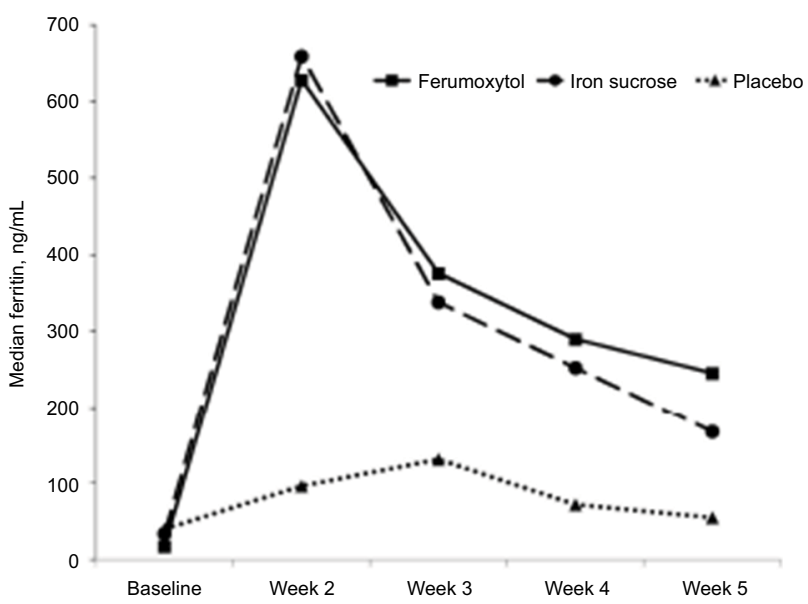

$\mathrm{mL}$, respectively, at week 2 , decreasing to $73.1 \mathrm{ng} / \mathrm{mL}$ and $65.2 \mathrm{ng} / \mathrm{mL}$, respectively, at week 5 .

Among the cancer subgroup, one patient in the IV ferumoxytol and one patient in the placebo group continued to receive treatment with an ESA (epoetin alfa) during the study. However, the doses remained unchanged throughout the study. Blood transfusions were received by $8 \%$ $(n=6)$ of the ferumoxytol-treated patients, $23 \%(n=3)$ of the iron sucrose-treated patients, and none of the placebotreated patients. Among the full study population, $1.4 \%$ of ferumoxytol-treated patients, $2.0 \%$ of iron sucrose-treated patients, and $2.5 \%$ of placebo-treated patients received transfusions.

Among the ferumoxytol treatment group the mean FACIT-F score was 29.0 at baseline. This increased 
significantly over baseline at all time points to 36.9 at week 5 $(P<0.0001$, paired $t$-test) (Figure 2B).

\section{Safety}

Summaries of the frequency and severity of AEs among patients in the cancer subgroup and the full study population are provided in Table 2. Overall rates of AEs and SAEs in the cancer subgroup; mirrored those reported in the overall study population. The overall rate of AEs was $63 \%$ among the cancer subgroup, the incidence of AEs was $64.0 \%$ for IV ferumoxytol. Given the small numbers of patients with cancer in the iron sucrose and placebo groups, comparisons regarding the relative rates of AEs are not appropriate. The large majority of events were not considered by investigators to be related to study treatment (Table 2).

SAEs were reported in $9.3 \%$ of patients receiving IV ferumoxytol, $7.7 \%$ receiving iron sucrose, and $10.0 \%$ receiving placebo in the cancer subgroup. This compares to rates of SAEs of $3.3 \%, 2.5 \%$, and $3.0 \%$, respectively, for IV ferumoxytol, iron sucrose, and placebo in the overall pooled study population. Two SAEs (hypersensitivity and anaphylactic reaction) in IV ferumoxytol-treated patients with cancer were considered drug related. There was one patient death in the ferumoxytol treatment group (duodenal obstruction secondary to pancreatic tumor) that was considered by the investigator to be unrelated to treatment. There was also one patient death in the placebo group (malignant lung neoplasm) which was considered by the investigator to be unrelated to treatment.

The most frequently occurring TEAEs are listed for the patients in the cancer subgroup and the pooled overall study population in Table 3. The most commonly occurring AEs among ferumoxytol-treated patients were abdominal pain, diarrhea, and nausea ( $5.3 \%$ for each) in the cancer subgroup, and headache (5.3\%), nausea (3.8\%), and dizziness (3.3\%) in the overall pooled study population.
Table 3 Treatment-emergent adverse events

\begin{tabular}{|c|c|c|c|}
\hline $\begin{array}{l}\text { AE preferred term, } \\
\text { n (\%) }\end{array}$ & Treatment grc & oups & \\
\hline \multicolumn{4}{|c|}{$\begin{array}{l}\text { TEAEs occurring in }>I \text { ferumoxytol-treated patient in the } \\
\text { cancer subgroup }\end{array}$} \\
\hline & $\begin{array}{l}\text { Ferumoxytol } \\
(n=75)\end{array}$ & $\begin{array}{l}\text { Iron sucrose } \\
(n=13)\end{array}$ & $\begin{array}{l}\text { Placebo } \\
(n=10)\end{array}$ \\
\hline Abdominal pain & $4(5.3)$ & $0(0.0)$ & $0(0.0)$ \\
\hline Diarrhea & $4(5.3)$ & $0(0.0)$ & $I(I 0.0)$ \\
\hline Nausea & $4(5.3)$ & I (7.7) & $0(0.0)$ \\
\hline Cough & $3(4.0)$ & $0(0.0)$ & $0(0.0)$ \\
\hline Dizziness & $3(4.0)$ & $0(0.0)$ & $0(0.0)$ \\
\hline Hypokalemia & $3(4.0)$ & $0(0.0)$ & $0(0.0)$ \\
\hline Leukopenia & $3(4.0)$ & $0(0.0)$ & $0(0.0)$ \\
\hline Neutropenia & $3(4.0)$ & $0(0.0)$ & $2(20.0)$ \\
\hline Vomiting & $3(4.0)$ & $0(0.0)$ & $0(0.0)$ \\
\hline Back pain & $2(2.7)$ & $0(0.0)$ & $0(0.0)$ \\
\hline Constipation & $2(2.7)$ & I (7.7) & $0(0.0)$ \\
\hline Dry mouth & $2(2.7)$ & $0(0.0)$ & $0(0.0)$ \\
\hline Dysgeusia & $2(2.7)$ & $0(0.0)$ & $0(0.0)$ \\
\hline Iron deficiency anemia & $2(2.7)$ & $0(0.0)$ & $0(0.0)$ \\
\hline \multicolumn{3}{|l|}{ progression } & $0(0.0)$ \\
\hline Pain in extremity & $2(2.7)$ & $0(0.0)$ & $0(0.0)$ \\
\hline Pyrexia & $2(2.7)$ & $0(0.0)$ & $I(10.0)$ \\
\hline Urinary tract infection & $2(2.7)$ & $0(0.0)$ & $0(0.0)$ \\
\hline
\end{tabular}

TEAEs occurring in $\geq 1.5 \%$ of ferumoxytol-treated patients in the pooled overall study population

\begin{tabular}{llll}
\hline & $\begin{array}{l}\text { Ferumoxytol } \\
(\mathbf{n}=1,014)\end{array}$ & $\begin{array}{l}\text { Iron sucrose } \\
(\mathbf{n}=1 \mathbf{9 9})\end{array}$ & $\begin{array}{l}\text { Placebo } \\
(\mathbf{n}=\mathbf{2 0 0})\end{array}$ \\
\hline Headache & $54(5.3)$ & $\mathrm{II}(5.5)$ & $12(6.0)$ \\
Nausea & $39(3.8)$ & $7(3.5)$ & $5(2.5)$ \\
Dizziness & $33(3.3)$ & $3(1.5)$ & $7(3.5)$ \\
Diarrhea & $21(2.1)$ & $0(0.0)$ & $6(3.0)$ \\
Urinary tract infection & $19(1.9)$ & $3(1.5)$ & $6(3.0)$ \\
Nasopharyngitis & $18(1.8)$ & $2(1.0)$ & $4(2.0)$ \\
Dysgeusia & $18(1.8)$ & $13(6.5)$ & $1(0.5)$ \\
Back pain & $17(1.7)$ & $1(0.5)$ & $2(1.0)$ \\
Vomiting & $16(1.6)$ & $4(2.0)$ & $2(1.0)$ \\
Abdominal pain & $15(1.5)$ & $3(1.5)$ & $5(2.5)$ \\
Fatigue & $15(1.5)$ & $2(1.0)$ & $3(1.5)$ \\
\hline
\end{tabular}

Abbreviations: AEs, adverse events; TEAEs, treatment-emergent adverse events.

Table 2 Adverse event summary: cancer subgroup and overall study population

\begin{tabular}{|c|c|c|c|c|c|c|}
\hline \multirow[t]{2}{*}{ AE category, n (\%) } & \multicolumn{3}{|c|}{ Cancer subgroup } & \multicolumn{3}{|c|}{ Pooled overall study population } \\
\hline & $\begin{array}{l}\text { Ferumoxytol } \\
(n=75)\end{array}$ & $\begin{array}{l}\text { Iron sucrose } \\
(n=\mid 3)\end{array}$ & $\begin{array}{l}\text { Placebo } \\
(n=10)\end{array}$ & $\begin{array}{l}\text { Ferumoxytol } \\
(n=1,0 \mid 4)\end{array}$ & $\begin{array}{l}\text { Iron sucrose } \\
(n=199)\end{array}$ & $\begin{array}{l}\text { Placebo } \\
(n=200)\end{array}$ \\
\hline All AEs & $48(64.0)$ & $6(46.2)$ & $8(80.0)$ & $467(46.1)$ & $88(44.2)$ & $86(43.0)$ \\
\hline Related $\mathrm{AEs}^{\mathrm{a}}$ & $6(8.0)$ & $0(0.0)$ & I (I0.0) & 147 (14.5) & $32(16.1)$ & I5 (7.5) \\
\hline Serious AEs & $7(9.3)$ & I (7.7) & I (I0.0) & $33(3.3)$ & $5(2.5)$ & $6(3.0)$ \\
\hline Related serious AEs & $2(2.7)$ & $0(0.0)$ & $0(0.0)$ & $6(0.6)$ & $0(0.0)$ & $0(0.0)$ \\
\hline AEs of special interest ${ }^{\mathrm{b}}$ & $4(5.3)$ & I (7.7) & $0(0.0)$ & $33(3.3)$ & $10(5.0)$ & $2(1.0)$ \\
\hline AEs resulting in study discontinuation & $0(0.0)$ & $0(0.0)$ & $0(0.0)$ & $6(0.6)$ & $2(1.0)$ & $2(1.0)$ \\
\hline Death $^{c}$ & I (I.3) & $0(0.0)$ & I $(I 0.0)$ & $3(0.3)$ & $0(0.0)$ & I (0.5) \\
\hline
\end{tabular}

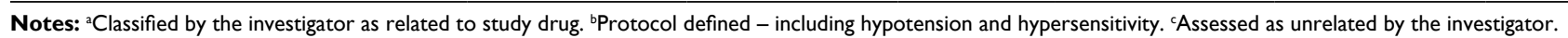
Abbreviation: AEs, adverse events. 


\section{Discussion}

This post hoc analysis focused on the subgroup of patients who had both IDA and cancer from two randomized, multicenter, global Phase III studies that evaluated the safety and efficacy of IV ferumoxytol in patients with a history of unsatisfactory oral iron therapy or in whom oral iron could not be used. The results indicate that cancer patients with IDA, who had not tolerated or responded to oral iron, responded to both IV ferumoxytol and iron sucrose with a significant increase from baseline in $\mathrm{Hgb}(1.8 \mathrm{~g} / \mathrm{dL}$ and $1.9 \mathrm{~g} / \mathrm{dL}$, respectively). Similar to the overall study population, the increase in $\mathrm{Hgb}$ was accompanied by a significant improvement in patientreported fatigue. Among the ferumoxytol treatment group, the lower bound CI of change from baseline (mean, 6.3; $95 \%$ CI $, 3.8,8.8) ; P<0.001$ ) was above the 3 -point minimum important difference for the FACIT-F scale, suggesting a clinically meaningful improvement.

The increase in mean $\mathrm{Hgb}$ at week 5 was somewhat lower in the current post hoc analysis of the pooled cancer patient subpopulation for patients receiving IV ferumoxytol (ie, $1.8 \mathrm{~g} / \mathrm{dL}$ ) than that observed for the total patient population in the original Studies 1 and $2(2.7 \mathrm{~g} / \mathrm{dL}$ in each). ${ }^{14,15}$ Similar results were seen for patients receiving iron sucrose with a smaller, but still substantial, mean increase in $\mathrm{Hgb}$ of $1.9 \mathrm{~g} / \mathrm{dL}$ from baseline to week 5 for the cancer patient subpopulation compared with an increase of $2.4 \mathrm{~g} / \mathrm{dL}$ for the total patient population in the original Study 2 publication. ${ }^{14}$

In general, cancer patients with anemia of inflammation $^{1}$ are expected to achieve a much smaller increase in $\mathrm{Hgb}$ than other patient groups. Notably, mean serum ferritin levels among those in the cancer subgroup receiving ferumoxytol were substantially higher at baseline $(121 \mathrm{ng} / \mathrm{mL})$ compared with levels in the overall populations in Study $1(22.6 \mathrm{ng} / \mathrm{mL})^{15}$ and Study $2(24.5 \mathrm{ng} / \mathrm{mL}){ }^{14}$ The higher baseline ferritin values in patients with cancer reflects the fact that ferritin is an acute-phase reactant that can be artificially elevated in inflammatory conditions and underscores its limited utility as a marker of IDA in patients with chronic disease.

For ferumoxytol-treated patients, the overall incidence of AEs was somewhat higher in the cancer subgroup compared with the pooled overall study population; however, treatment-related AEs tended to be lower among those in the cancer subgroup. There were too few patients in the cancer subgroup receiving either iron sucrose or placebo to make meaningful comparisons regarding relative rates of AEs between treatment groups. There was a higher incidence of serious AEs overall in the cancer subgroup, although most of these events were not considered related to study treatment. Similarly, a slightly greater proportion of patients with cancer receiving ferumoxytol or iron sucrose had AEs of special interest, defined as hypotension or hypersensitivity, compared with patients in the pooled overall study population, although the absolute numbers were small. Rates of individual AEs for patients receiving IV ferumoxytol were generally similar between the cancer subgroup and the pooled overall study population, with abdominal pain, diarrhea, and nausea (all 5.3\%) most common in the cancer subgroup and headache, nausea, and dizziness $(5.3 \%, 3.8 \%$, and $3.3 \%$, respectively) most common in the pooled overall study population.

Although ESAs are recommended for the treatment of CIA, only $40 \%-70 \%$ of patients have been shown to respond to ESAs with a substantial increase in Hgb. ${ }^{16}$ In addition, ESA therapy is associated with an increased risk of developing venous thromboembolism. ${ }^{17}$ The mechanism underlying this increased risk has not been established; however, results from a study by Henry et al suggest that ESA-associated venous thromboembolism in patients with CIA may be related, at least in part, to the thrombocytosis caused by ESA-induced iron-restricted erythropoiesis, and that this effect may be countered by IV iron supplementation. ${ }^{18}$

Several clinical trials have shown that IV iron improves response rates when coadministered with an ESA and may avoid iron-restricted erythropoiesis. ${ }^{16,19,20}$ However, there are few randomized studies evaluating the use of IV iron monotherapy in patients with anemia and cancer. Two randomized studies evaluated the use of iron monotherapy (iron sucrose) in patients with anemia and gynecologic cancer; however, these were very small, including a total of 52 patients receiving iron therapy. ${ }^{21,22}$ In one study, treatment with IV iron sucrose was shown to reduce the incidence of red blood cell transfusion among patients with anemia and gynecologic cancer receiving platinum-based chemotherapy compared to patients receiving oral iron (5/22 vs $14 / 22$ patients $[22.7 \%$ vs $63.6 \%$ ], respectively; $P<0.05) .{ }^{22}$ Significantly higher mean $\mathrm{Hgb}$ and hematocrit levels were reported after the first week of treatment among patients receiving IV iron sucrose compared with those receiving oral iron therapy, which persisted until the next cycle of chemotherapy. Similar results were shown in an earlier study evaluating monotherapy with IV iron sucrose in 30 patients with anemia and cervical cancer undergoing chemoradiotherapy compared with 45 patients receiving no iron therapy. ${ }^{21}$ In that study, administration of IV iron sucrose resulted in reduced red blood cell transfusions $(P=0.04)$ and 
reduced mean transfusion volume $(P=0.04)$. A randomized controlled trial comparing the efficacy and safety of IV iron isomaltoside with oral ferrous sulfate in patients with cancer and anemia found that iron isomaltoside was noninferior to oral iron in change in $\mathrm{Hgb}$ concentration from baseline to week 4 (difference estimate $0.016,95 \% \mathrm{CI}-0.26$ to 0.29 , $P<0.001$ ), with no significant difference in the proportion of patients experiencing an adverse event noted between treatment groups (72\% [165/229] in the iron isomaltoside group vs $70 \%$ [78/112] in the oral iron group). ${ }^{23}$ Results from the current study provide additional evidence of the efficacy and safety of IV iron therapy in cancer populations.

Limitations of this study include the retrospective design and the small number of patients in the iron sucrose and placebo groups, which limits the ability to draw any comparative conclusions. A strength of this study is that the vast majority of patients (98\%) did not receive concomitant ESAs. While none of the published clinical trials of IV iron in cancer-related anemia have shown any increase in tumor progression among patients receiving IV iron treatment, long-term follow-up data are still lacking. Larger, longer term studies are needed to confirm the efficacy and safety results and further define the responses achievable with iron monotherapy.

\section{Conclusion}

The results of the current study indicate that cancer patients with IDA, who had not tolerated or responded to oral iron, respond to both IV ferumoxytol and iron sucrose with a significant increase in Hgb. Based on these findings, IV iron monotherapy may offer a potentially effective treatment option for the management of IDA in patients with cancer who do not respond to or cannot tolerate oral iron therapy

\section{Acknowledgments}

Both the primary study and the writing of the secondary analysis were supported financially by AMAG Pharmaceuticals, Inc. Maria McGill, RPh, CMPP, of inScience Communications, Springer Healthcare (Philadelphia, PA, USA), and Bret Fulton, RPh, provided medical writing support funded by AMAG Pharmaceuticals, Inc.

\section{Disclosure}

SV-R. received research funding to support the clinical trial. NVD, WES, KB, and ZL are all employees of AMAG Pharmaceuticals, Inc., and hold equity in the company. The authors report no other conflicts of interest in this work.

\section{References}

1. National Comprehensive Cancer Network (NCCN). NCCN Clinical Practice Guidelines: Cancer- and Chemotherapy-Induced Anemia. Version 2.2017. NCCN; 2017. Available from: https://www.nccn.org/ store/login/login.aspx?ReturnURL=http://www.nccn.org/professionals/ physician_gls/pdf/anemia.pdf. Accessed October 8, 2017.

2. Knight K, Wade S, Balducci L. Prevalence and outcomes of anemia in cancer: a systematic review of the literature. Am J Med. 2004;116 Suppl 7A:11S-26S.

3. Schwartz RN. Anemia in patients with cancer: incidence, causes, impact, management, and use of treatment guidelines and protocols. Am J Health Syst Pharm. 2007;64(3 Suppl 2):S5-S13.

4. Steensma DP. Is anemia of cancer different from chemotherapy-induced anemia? J Clin Oncol. 2008;26(7):1022-1024.

5. Wilson J, Yao GL, Raftery J, et al. A systematic review and economic evaluation of epoetin alpha, epoetin beta and darbepoetin alpha in anaemia associated with cancer, especially that attributable to cancer treatment. Health Technol Assess. 2007;11(13):1-202, iii-iv.

6. Perazella MA. Onco-nephrology: renal oxicities of chemotherapeutic agents. Clin J Am Soc Nephrol. 2012;7(10):1713-1721.

7. Wood PA, Hrushesky JM. Cisplatin-associated anemia: an erythropoietin deficiency syndrome. J Clin Invest. 1995;95(4):1650-1659.

8. Barni S, Cabiddu M, Guarneri P, Lonati V, Petrelli F. The risk for anemia with targeted therapies for solid tumors. Oncologist. 2012;17(5):715-724.

9. Gafter-Gvili A, Rozen-Zvi B, Vidal L, Leibovici L, Vansteenkiste J, Gafter U, Shpilberg O. Intravenous iron supplementation for the treatment of chemotherapy-induced anaemia - systematic review and meta-analysis of randomised controlled trials. Acta Oncol. 2013;52(1): $18-29$.

10. Rosner MH, Auerbach M. Ferumoxytol for the treatment of iron deficiency. Expert Rev Hematol. 2011;4(4):399-406.

11. Jahn MR, Andreasen HB, Fütterer S, et al. A comparative study of the physiochemical properties of iron isomaltoside 100 (Monofer), a new intravenous iron preparation and its clinical implications. Eur J Pharm Biopharm. 2011;78(3):4880-4491.

12. Pai AB, Garba AO. Ferumoxytol: a silver lining in the treatment of anemia of chronic kidney disease or another dark cloud? J Blood Med. 2012;3:77-85.

13. Feraheme (ferumoxytol) injection: US [prescribing information]. Waltham, MA: AMAG Pharmaceuticals, Inc.; 2015.

14. Hetzel D, Strauss W, Bernard K, Li Z, Urboniene A, Allen LF. A phase III, randomized, open-label trial of ferumoxytol compared with iron sucrose for the treatment of iron deficiency anemia in patients with a history of unsatisfactory oral iron therapy. Am J Hematol. 2014;89(6):646-650.

15. Vadhan-Raj S, Strauss W, Ford D, Bernard K, Boccia R, Li J, Allen LF. Efficacy and safety of IV ferumoxytol for adults with iron deficiency anemia previously unresponsive to or unable to tolerate oral iron. $\mathrm{Am}$ J Hematol. 2014;89(1):7-12.

16. Hedenus M, Birgegard G. The role of iron supplementation during epoietin treatment for cancer-related anemia. Med Oncol. 2009;26(1):105-115.

17. Bennett CL, Silver SM, Djulbegovic B, et al. Venous thromboembolism and mortality associated with recombinant erythropoietin and darbepoetin administration for the treatment of cancer-associated anemia. JAMA. 2008;299(8):914-924.

18. Henry DH, Dahl NV, Auerbach MA. Thrombocytosis and venous thromboembolism in cancer patients with chemotherapy induced anemia may be related to ESA induced iron restricted erythropoiesis and reversed by administration of IV iron. Am J Hematol. 2012;87(3):308-310.

19. Henry DH, Dahl NV, Auerbach M, Tchekmedyian S, Laufman LR. Intravenous ferric gluconate significantly improves response to epoetin alfa versus oral iron or no iron in anemic patients with cancer receiving chemotherapy. Oncologist. 2007;12(2):231-242. 
20. Auerbach M, Ballard H, Trout JR, et al. Intravenous iron optimizes the response to recombinant human erythropoietin in cancer patients with chemotherapy-related anemia: a multicenter, open-label, randomized trial. J Clin Oncol. 2004;22(7):1301-1307.

21. Kim YT, Kim SW, Yoon BS, et al. Effect of intravenously administered iron sucrose on the prevention of anemia in the cervical cancer patients treated with concurrent chemoradiotherapy. Gynecol Oncol. 2007;105(1):199-204.
22. Dangsuwan P, Manchana T. Blood transfusion reduction with intravenous iron in gynecologic cancer patients receiving chemotherapy. Gynecol Oncol. 2010;116(3):522-525.

23. Birgegård G, Henry D, Glaspy J, Chopra R, Thomsen LL, Auerbach M. A randomized noninferiority trial of intravenous iron isomaltoside versus oral iron sulfate in patients with nonmyeloid malignancies and anemia receiving chemotherapy: The PROFOUND Trial. Pharmacotherapy. 2016;36(4):402-414. 


\section{Supplementary material}

Names of each approving institutional review board and ethics committee

- Ajou University Hospital Institutional Review Board SF, annex, Ajou University Hospital, Korea;

- Arrowhead Regional Medical Center Institutional Review Board, USA;

- Bellberry Human Research Ethics Committee, Australia;

- Centre For Digestive Diseases Human Research Ethics Committee, Australia;

- Christiana Care Health Services Institutional Review Board, USA;

- Columbus Regional Healthcare System, USA;

- Comitato Etico per la Sperimentazione Azienda Ospedaliera - Universita di Padova, Italy;

- Comite de Protection des Personnes Ile de France X, France;

- Comite Etico de Investigacion Clinica Centro Medico Teknon, Spain;

- Dispur Hospitals Ethics Committee, India;

- Ethics Committee for Clinical Research at Development Society of Pauls Stradins Clinical University Hospital, Latvia;

- Ethics Committee Kodlikeri Memorial Hospital (K.M.H) \& CIIGMA Hospital, India;

- Ethics Committee of Bangalore Medical College \& Research Institute and Associated Hospitals, India;

- Ethics Committee Poona Hospital and Research Centre Poona Hospital and Research Centre, India;

- Ethics Committee, Lifeline Multi Specialty Hospital No 5/639 Rajiv Gandhi Salai (OMR), India;

- G. Kuppuswamy Naidu Memorial Hospital Institutional Ethics Committee, India;

- Gachon University Gil Hospital Institutional Review Board, Korea;

- Gangnam Severance Hospital Institutional Review Board, Korea;

- Global Health Concern Ethics Committee, India;

- Horizon Health Network Research Ethics Board, Canada;

- Human Research Protection Program, 504 Oxford House, USA;

- Inha University Hospital Institutional Review Board, Korea;

- Institutional Ethics Committee - Global Hospitals Lakdika-pool, India;
- Institutional Ethics Committee, Andhra Medical College, India;

- Institutional Ethics Committee, Chhatrapati Shahuji Maharaj Medical University, India;

- Institutional Ethics Committee, Kasturba Medical College, India;

- Institutional Ethics Committee, Krishna Institute of Medical Sciences, India;

- Institutional Ethics Committee, M.V. Hospital \& Research Centre, India;

- Institutional Ethics Committee, Sri Ramachandra University, India;

- Institutional Review Board of Asan Medical Center, Korea;

- Institutional Review Board of Ewha Womans University Mokdong Hospital, Korea;

- Institutional Review Board of Kangdong Sacred Heart Hospital, Korea;

- Keimyung University Dongsan Hospital Institutional Review Board, Korea;

- Komisja Bioetyczna przy Okregowej Izbie Lekarskiej w Bialymstoku, Poland;

- Landesaerztekammer Rheinland-Pfalz Koerperschaft des Oeffentlichen Rechts, Germany;

- Lithuanian Bioethics Committee, Lithuania;

- M.S. Ramaiah Medical College and Teaching Hospital Ethical Review Board, India;

- Manavata Clinical Research Institute Professional Ethics Committee, India;

- Medical Research Council Ethics Committee for Clinical Pharmacology, Hungary;

- Meenakshi Mission Hospital and Research Centre Ethical Review Board, India;

- Melbourne Health Human Research Ethics Committee, Australia;

- Meridian Health Institutional Review Board, USA;

- National Ethics Committee for Clinical Study of Medicine, Romania;

- New York Downtown Hospital IRB, USA;

- Nottingham Research Ethics Committee 2, UK;

- Pharma Ethics (Pty) Ltd, South Africa;

- Royal Adelaide Hospital Research Ethics Committee, Australia;

- S.R. Kalla Memorial Ethical Committee for Human Research, India;

- Samsung Medical Center Institutional Review Board, Korea; 
- SEAROC Ethics Committee, SEAROC Cancer Center, S.K. Soni Hospital, India;

- Seoul National University Hospital Institutional Review Board, Korea;

- Soonchunhyang University Cheonan Hospital Institutional Review Board, Korea;

- Sydney West Area Health Service Human Research Ethics Committee - Concord Repatriation General Hospital (CRGH Zone), Australia;
- The Catholic University of Korea, St. Vincent's Hospital Institutional Review Board, Korea;

- The Central Ethics Commission of Ministry of Health of Ukraine, Ukraine;

- The University of Texas M.D. Anderson Cancer Center Office of Protocol Research - Unit 198, USA;

- Western Institution Review Board, Inc. (WIRB), USA.
Journal of Blood Medicine

\section{Publish your work in this journal}

The Journal of Blood Medicine is an international, peer-reviewed, open access, online journal publishing laboratory, experimental and clinical aspects of all aspect pertaining to blood based medicine including but not limited to: Transfusion Medicine; Blood collection, Donor issues, Transmittable diseases, and Blood banking logistics; Immunohematology; Artificial and alternative
Dovepress

blood based therapeutics; Hematology; Biotechnology/nanotechnology of blood related medicine; Legal aspects of blood medicine; Historical perspectives. The manuscript management system is completely online and includes a very quick and fair peer-review system. Visit http://www.dovepress.com/ testimonials.php to read real quotes from published authors. 